\title{
miR-34b-a novel plasma marker for Huntington disease?
}

New research suggests that plasma levels of a microRNA (miRNA) molecule, miR-34b, could be a useful biomarker of disease progression in patients with Huntington disease (HD) - even before the onset of clinical symptoms. "To our knowledge, this is the first identification of an miRNA associated with the preclinical manifestation of HD that is detectable outside of the nervous system," asserts Maria Björkqvist, the study's corresponding author.

The researchers initially used a neuronalcell-based assay to identify two miRNAs that showed upregulated expression in the presence of mutant huntingtin protein. The mutant protein causes manifestations of HD through its ability to modulate gene transcription and translation.

After confirming the stability of the two miRNAs to repeated freeze-thaw cycles, the team measured levels of the miRNAs in stored frozen plasma samples from 27 patients with HD (either with or without clinical symptoms) and 12 age-matched controls. Only miR-34b
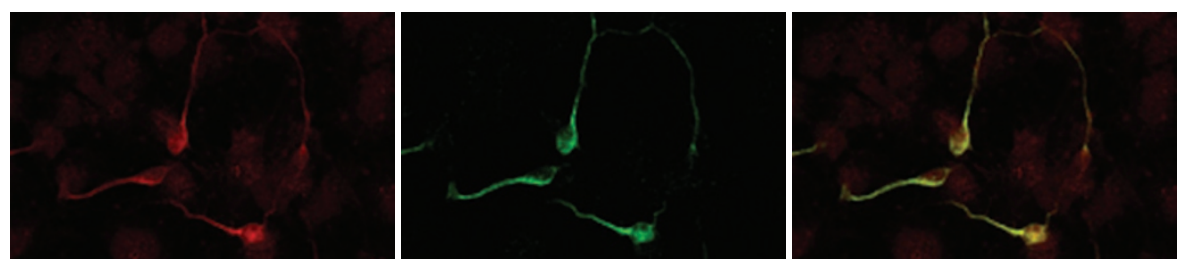

Neurons (green) engineered to express mutant huntingtin protein (red). Courtesy of Dr Philip Gaughwin.

levels were significantly elevated in samples from asymptomatic patients. Furthermore, expression of miR-34b showed a linear correlation with levels of mutant huntingtin protein in both neuronal cells and pluripotent cells engineered to express this protein, although the cytotoxic effects of mutant huntingtin were only observed in differentiated neurons.

"Our observations raise the intriguing hypothesis that common, mutant huntingtin-interacting products of somatic cell transcriptomes mediate mutant huntingtin-induced cytotoxicity, and that these products are absent or functionally sequestered from mutant huntingtin in pluripotent cells," comments Björkqvist.
In their report, Björkqvist and colleagues acknowledge that their results are based on a small patient cohort, and that "additional observations in larger patient numbers are required to establish the biomarker potential of miR-34b." The researchers plan to replicate their findings and to monitor miR-34b levels over time in a larger group of patients with $\mathrm{HD}$, as well as conducting in vitro studies on the effects of miR-34b dysregulation.

\section{Vanisha Parekh}

Original article Gaughwin, P. M. et al. Hsa-miR-34b is a plasma-stable microRNA that is elevated in pre-manifest Huntington's disease. Hum. Mol. Genet. doi:10.1093/ $\mathrm{hmg} / \mathrm{ddr} 111$ 